Cahiers de philosophie de l'université de

Totalitas. Aux origines d'un concept

\title{
Body and Soul. Pierre de Jean Olivi sur la totalité et l'hylémorphisme
}

\section{Christian Rode}

\section{(2) OpenEdition \\ 1 Journals}

Édition électronique

URL : https://journals.openedition.org/cpuc/346

DOI : $10.4000 /$ cpuc.346

ISSN : 2677-6529

Éditeur

Presses universitaires de Caen

\section{Édition imprimée}

Date de publication : 31 décembre 2016

Pagination : 133-152

ISBN : 978-2-84133-842-9

ISSN : $1282-6545$

Référence électronique

Christian Rode, "Body and Soul. Pierre de Jean Olivi sur la totalité et l'hylémorphisme », Cahiers de philosophie de l'université de Caen [En ligne], 53 | 2016, mis en ligne le 01 février 2019, consulté le 01 février 2023. URL : http://journals.openedition.org/cpuc/346 ; DOI : https://doi.org/10.4000/cpuc.346

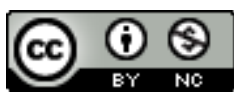

Creative Commons - Attribution - Pas d'Utilisation Commerciale 4.0 International - CC BY-NC 4.0 https://creativecommons.org/licenses/by-nc/4.0/ 


\title{
Body and Soul. Pierre de Jean Olivi sur la totalité et l'hylémorphisme ${ }^{1}$
}

\begin{abstract}
Q UELLE EST LA PRÉSENCE DE L’ÂME AU CORPS? Comment sont-ils connectés? La philosophie pose ces questions depuis Platon ${ }^{2}$ et Aristote. Chez ce dernier, certains extraits suggèrent fortement que l'âme existe comme un tout dans chacune des parties du corps. Par exemple, dans le De anima (en I 5, 411b14-27, ainsi qu'en II 2, 413b18-29), le Stagirite argumente contre l'opinion selon laquelle une partie de l'âme informe une partie du corps. Au contraire selon Aristote, dans chaque partie du corps, toutes les parties de l'âme, c'est-à-dire l'âme en tant que tout, est présente 3 . Il illustre son propos par l'exemple d'une plante ou d'un insecte coupé en deux moitiés, lesquelles peuvent continuer à vivre après l'opération. Ces moitiés n'auraient pu survivre si l'âme entière avec toutes ses fonctions (comme les puissances de se mouvoir et de sentir) ne les informait pas. Plotin ${ }^{4}$ et Augustin ${ }^{5}$ soutiennent explicitement la thèse selon laquelle l'âme entière est présente dans chaque partie du corps, et Thomas d'Aquin le reprend au $\mathrm{XIII}^{\mathrm{e}}$ siècle, modifiant la thèse de son maître Albert le Grand ${ }^{6}$. L'objet de mon article est la critique que fait Pierre de Jean Olivi de ce type de thèse. Lorsqu'il traite de l'unité de l'âme et du corps, tant Thomas d'Aquin que Pierre de Jean Olivi emploient la notion de totalitas. L'usage différent que ces penseurs font de ce concept met en lumière les différences sous-jacentes
\end{abstract}

1. Je remercie Maud Pouradier pour son excellente traduction. Toutefois, je reste seul responsable des éventuelles erreurs de cet article.

2. Par exemple Plato, Phaedo, J. Burnet (éd.), Oxford, Clarendon Press, 1959.

3. Aristote, De generatione animalium II 1, 734a14 sq.

4. Plotin, Enn. IV , 2 (4), 1; IV, 3 (27), 22, in Plotini opera, t. II, P. Henry et H.-R. Schwyzer (éd.), Paris - Bruxelles, Desclée de Brouwer - Éd. Universelle, 1959, p. 3 sq., p. 46 sq.

5. Augustin, De trinitate VI, 6, 8, W. J. Mountain et F. Glorie (éd.), Turnhout, Brepols, 1968, p. 237 .

6. Albert le Grand, De homine I 3, De coniunctione animae et corporis, $\mathrm{H}$. Anzulewicz et J. Söder (éd.), Münster, Aschendorff (Opera omnia, 27, 2), 2008, p. 570-572. 
dans leurs psychologies respectives. Je montrerai que Thomas d'Aquin traite principalement de l'unité de quelque chose de simple (l'âme en tant qu'unique forme substantielle de l'être humain) et de la diversité corporelle, tandis que Pierre de Jean Olivi, dans le cadre conceptuel d'une pluralité de formes substantielles, s'intéresse à une relation d'ordre supérieur: une relation de relations 7 .

En critiquant Thomas d'Aquin, Pierre de Jean Olivi s'attaque à un point faible de la psychologie thomiste: d'un côté l'âme est un principe de vie étroitement connecté au corps humain ${ }^{8}$, et de l'autre l'âme intellective a un esse propre, une subsistance, et peut survivre à la corruption du corps ${ }^{9}$. Mais pour Olivi la thèse thomiste d'une présence essentielle - et tout à fait singulière - de l'âme au corps ne peut rendre compte à la fois de l'information du corps par l'âme et de la subsistance de l'âme. Par conséquent, Olivi abandonne le holenmérisme de l'Aquinate, c'est-à-dire la théorie selon laquelle l'âme humaine est essentiellement présente comme un tout dans chaque partie du corps. Le traitement olivien de la totalité aboutit à une conception anti-aristotélicienne de l'intellect: un être susceptible de degrés et de mouvement, au même titre que les corps physiques de changement. Que l'anima intellectiva soit ainsi automotrice, et par là libre, est au cœur d'une éthique volontariste.

Je commencerai par présenter la thèse thomiste, puis j'exposerai les arguments que Pierre de Jean Olivi lui oppose lorsqu'il s'agit de savoir si les formes substantielles sont susceptibles du plus et du moins (magis et minus). Je conclurai par la solution proprement olivienne et par l'évaluation systématique de ses avantages et de ses inconvénients.

\section{Thomas d'Aquin}

Pour Thomas d'Aquin, il n'y a qu'une seule forme substantielle du corps humain: l'anima intellectiva ${ }^{10}$. Cependant, elle présente différentes fonctions ou puissances: la partie végétative - responsable de la nutrition, de la croissance et de la reproduction, la partie sensitive - principe du sens

7. Sur les différentes positions s'affrontant sur cette question au XIII e siècle, voir R. C. Dales, The Problem of the Rational Soul in the Thirteenth Century, Leyde - New York-Cologne, Brill, 1995.

8. Cf. note 10.

9. Thomas d'Aquin, Summa theologiae I, q. 75, a. 2, Editio Leonina, t. V, Rome, Typographia Polyglotta s. c. de propaganda fide, 1889, p.196a : «Respondeo dicendum quod necesse est dicere id quod est principium intellectualis operationis, quod dicimus animam hominis, esse quoddam principium incorporeum et subsistens».

10. Ibid., p. 208-210. 
perceptif - et la partie intellective - principe de la pensée et de la cognition. L'Aquinate adopte pour l'âme un modèle inclusiviste: une forme ontologiquement simple (l'âme intellective) inclut les différentes fonctions des âmes des animaux et des plantes, de même qu'une figure géométrique complexe comme un rectangle inclut des figures plus simples comme les triangles ${ }^{11}$. Ainsi, la simplicité ontologique de l'âme humaine est opposée à sa différentiation fonctionnelle ${ }^{12}$. Thomas d'Aquin prend en compte cette opposition en répondant à la question de savoir comment l'âme est unie au corps, et si elle est présente comme un tout dans chaque partie du corps. De manière générale pour le docteur angélique, l'âme est présente comme une totalité dans chaque partie, et cela parce que l'âme n'est pas simplement le moteur du corps. Si tel était le cas en effet, elle ne s'unirait qu'à une seule partie du corps, par exemple le cœur comme Albert le Grand l'a soutenu ${ }^{13}$. Au contraire pour Thomas d'Aquin, l'âme est la forme substantielle du corps, et une forme susbstantielle constitue ou configure l'être des parties de sa matière, à l'inverse d'une forme accidentelle, comme la forme d'une maison par exemple, qui participe seulement à la composition ou à l'ordonnancement de parties matérielles capables d'exister par elles-mêmes. Ne resteraient que les briques d'une maison, elles peuvent continuer d'exister. Mais les parties matérielles d'un être humain sont incapables d'exister en tant que (qua) parties humaines si l'organisme est détruit et si l'âme est séparée du corps. Comme Aristote l'a affirmé, une main séparée ne peut être appelée humaine que de manière équivoque ${ }^{14}$. C'est pourquoi la forme substantielle d'un être humain est présente comme un tout dans chaque partie du corps, puisqu'aucune partie du corps ne peut remplir sa fonction séparée de l'organisme et de sa forma substantialis ${ }^{15}$.

11. Thomas d'Aquin, Quodl. XI, q. 5, Editio Leonina, t. XXV, 1, Rome - Paris, Commissio Leonina - Cerf, 1996, p.158f.; De unitate intellectus, c. 1, Editio Leonina, t. XLIII, Rome, Editori di San Tommaso, 1976, p. 300.

12. E. Stump, Aquinas, Londres - New York, Routledge, 2003, p. 202. Cf. Thomas d'Aquin, Quaestiones disputatae de anima, q. 10, ad 18, B.-C. Bazán (éd.), Editio Leonina, t. XXIV, 1, Rome - Paris, Commissio Leonina - Cerf, Paris, 1996, p. 94b; Quaestiones disputatae de anima, q. 9, ad 14, p. 85a-b: «Ad quartum decimum dicendum quod licet anima sit forma simplex secundum essentiam, est tamen multiplex virtute, secundum quod est principium diuersarum operationum».

13. Albert le Grand, De homine..., p. 572.

14. Aristote, De generatione animalium I 19, 726b22 sq., II 1, 734a15, b24, II 1, 735a7 sq.

15. Thomas d'Aquin, Summa theologiae..., I, q. 76, a. 8, p. 232 sq.: "Sed quia anima unitur corpori ut forma, necesse est quod sit in toto, et in qualibet parte corporis. Non enim est forma corporalis accidentalis, sed substantialis. Substantialis autem forma non solum est perfectio totius, sed cuiuslibet partis. Cum enim totum consistat ex partibus, forma totius quae non dat esse singulis partibus corporis, est forma quae est compositio et ordo, sicut forma domus: et talis forma est accidentalis. Anima vero est forma substantialis: unde oportet quod sit 
Aussi l'Aquinate argumente-t-il de manière fonctionnaliste pour prouver la thèse ontologique selon laquelle l'âme est présente comme un tout dans chaque partie du corps.

C'est à ce moment de l'argumentation qu'il introduit la notion de totalité: pour lui, il y a trois manières d'être un tout (totalitas), c'est-à-dire une triple manière d'être divisible en parties: premièrement, il y a un tout quantitatif qui consiste en parties quantitatives, par exemple une ligne ou une figure géométrique. L'âme humaine ne peut avoir une telle sorte de totalité, puisqu'elle est immatérielle et simple. Deuxièmement, il y a une totalité essentielle ou une totalité de perfection. Par exemple la définition d'une essence, disons d'un homme, comprend des parties de définition, en l'occurrence «animal» et «rationnel»; ou encore une substance composée est faite de forme et de matière. Troisièmement, Thomas d'Aquin conçoit un tout potentiel, qui consiste en parties virtuelles, c'est-à-dire en plusieurs puissances ou fonctions. La question est alors la suivante: quelle sorte de totalité peut être attribuée à l'âme humaine, ou à la forme substantielle en général ? La première peut être exclue puisque l'âme humaine immatérielle n'a aucune quantité, qu'elle soit substantielle ou accidentelle. L'âme humaine possède en revanche la seconde et la troisième sortes de totalité ${ }^{16}$.

Mais si nous demandons selon laquelle de ces deux dernières totalités le tout qu'est l'âme existe dans chaque partie du corps, Thomas d'Aquin répond: seulement selon la totalité essentielle ou perfective. En effet, si nous considérons la totalité virtuelle, force est de conclure que seule une partie du tout potentiel est présente dans une partie du corps (par exemple la puissance de voir seulement dans les yeux, la puissance d'entendre seulement dans les oreilles, etc.). Et il y a même une puissance qui n'est présente dans aucun organe du tout: la puissance intellective. Ainsi, d'un point de vue ontologique, l'âme est présente comme un tout dans chaque partie corporelle et constitue l'être de chaque organe, mais si nous adoptons une perspective fonctionnaliste, toutes les fonctions ou puissances de l'âme ne sont pas simultanément présentes comme un tout dans chaque partie du corps ${ }^{17}$.

En distinguant ces deux perspectives - ontologique et fonctionnaliste Thomas d'Aquin concilie deux visions aristotéliciennes traditionnelles de l'âme, qui présentent entre elles une tension certaine: d'un côté on conçoit

forma et actus non solum totius, sed cuiuslibet partis. Et ideo, recedente anima, sicut non dicitur animal et homo nisi aequivoce, quemadmodum et animal pictum vel lapideum; ita est de manu et oculo, aut carne et osse, ut Philosophus dicit».

16. Thomas d'Aquin, Summa theologiae..., I, q. 76, a. 8, p. 232b.

17. Ibid., I, q. 76, a. 8, p. 233a: «Sed quia anima totalitatem quantitativam non habet, nec per se nec per accidens, ut dictum est; sufficit dicere quod anima tota est in qualibet parte corporis secundum totalitatem perfectionis et essentiae, non autem secundum totalitatem virtutis». 
l'âme comme une forme constituant l'être particulier du corps, c'est-à-dire comme ce qui informe la matière corporelle. En ce sens, l'âme est le principe de vie d'un organisme matériel, et elle influence l'être des parties du corps ${ }^{18}$. D'un autre côté, il faut entendre par «âme» l'intellect, c'est-à-dire quelque chose qui est introduit thyraten (du dehors), qui est extérieur à l'organisation matérielle d'un être vivant et qui en est ontologiquement séparable ${ }^{19}$. On trouve déjà ces deux caractérisations de l'âme chez Aristote, et Thomas d'Aquin prend soin d'éviter tout conflit direct entre elles dans sa psychologie. Par exemple, dans son attaque du monopsychisme averroïste dans le De unitate intellectus, il définit l'intellect comme une puissance immatérielle (virtus) de l'âme qui est la forme d'un corps physique et organique ${ }^{20}$. En ce qui concerne les fonctions de l'âme, l'intellect n'a pas d'organe et n'est pas directement connecté au corps, mais sa puissance spirituelle et ses activités appartiennent à l'âme qui, ontologiquement parlant, est la forme substantielle du corps. Par conséquent, il n'est pas permis de dire que l'intellect connaît, comme le soutient Averroès, mais seulement que cet être humain particulier pense ${ }^{21}$.

Le holenmérisme thomiste (la thèse selon laquelle le tout de l'âme est présent dans chaque partie du corps) est une option théorique recevable aux deux conditions suivantes: 1 ) Nous pouvons différencier une perspective ontologique d'une perspective fonctionnaliste sur l'âme 2) Il n'y a pas de contradiction entre ces deux perspectives, qui peuvent être conciliées dans une psychologie unifiée. La critique de Pierre de Jean Olivi commence précisément ici, en ce qu'il tente de montrer que la doctrine d'une unique forme substantielle conduit à des contradictions. Il rejette donc le holenmérisme et choisit à la place la thèse d'une pluralité des formes.

\section{La critique de Pierre de Jean Olivi}

Avant d'entrer plus avant dans le détail de la critique olivienne, il faut considérer plus largement ses différences terminologiques avec l'Aquinate. Comme Thomas d'Aquin, Pierre de Jean Olivi suppose que l'âme humaine rationnelle est simple parce qu'elle est spirituelle, mais il introduit une hiérarchie de degrés ontologiques de simplicité: le plus haut degré de simplicité est la simplicité de Dieu, excluant quelque structure interne que

18. Aristote, De anima II 1, 412a27-b9.

19. Aristote, De generatione animalium II, 3, 736b27-29, De anima III 4, 429a10-b5, III 5, 430a17-25.

20. Thomas d'Aquin, De unitate intellectus contra Averroistas, Editio Leonina, t. XLIII, Rome, Editori di San Tommaso, 1976, c. 1, p. 296b-297a, p.301a, c.3, p.307a, c. 4, p.307b.

21. Ibid., c.3, p.303a. 
ce soit et allant de pair avec l'infinité. Le degré intermédiaire de simplicité appartient à l'âme humaine ou aux autres créatures spirituelles, comme les anges. Cette sorte de simplicité autorise explicitement une grandeur interne, ou une structure de parties essentielles distinctes, ou un plus ou moins d'intension essentielle, mais aucune extension quantitative que ce soit. Le plus bas degré de simplicité est celui des points géométriques. Il ne s'agit que d'une simplicité extensive et quantitative - c'est-à-dire une absence complète d'extension - bien qu'aucune propriété spirituelle n'y soit liée ${ }^{22}$.

Un autre choix terminologique doit être expliqué: la formulation totum et totaliter que Pierre de Jean Olivi utilise souvent lorsqu'il argumente contre Thomas d'Aquin. Quelle est la différence entre une forme substantielle présente à un corps comme un tout d'une part (totum) et complètement d'autre part (totaliter) ? Olivi décrit la différence dans une question traitant de la présence de l'espèce (species) dans les individus qui lui appartiennent. La species est présente comme un tout dans chaque individu puisque chaque individu est un homme ou un âne, c'est-à-dire est subsumé sous la species correspondante. Mais la species n'est pas présente à un individu totaliter. Autrement dit, sa présence n'est pas restreinte à un seul individu, mais a un ambitus, c'est-à-dire une extension, qui couvre l'ensemble des individus existant lui correspondant. Cet ambitus n'est rien de réel, mais un simple mode de cognition ${ }^{23}$ : l'universel a seulement une existence intra-mentale. Olivi est en effet opposé à toute forme de réalisme des universaux ${ }^{24}$.

Le philosophe formule sa critique de Thomas d'Aquin dans la question $22 \mathrm{du}$ second livre de son commentaire des Sentences. Le texte traite de l'intension et de la rémission des formes substantielles. Autrement dit,

22. Pierre de Jean Olivi, Quaestiones in secundum librum Sententiarum, q. 49, B. Jansen (éd.), vol. II, q. 49-71, Quaracchi, Collegium S. Bonaventurae (Bibliotheca Franciscana Scholastica Medii Aevi, 5), 1924, p.17 sur la simplicité quantitative et la simplicité de la créature spirituelle. Cf. Pierre de Jean Olivi, Quaestiones in secundum librum Sententiarum, q. 34, B. Jansen (éd.), vol. I, q. 1-48, Quaracchi, Collegium S. Bonaventurae (Bibliotheca Franciscana Scholastica Medii Aevi, 4), 1922, p.607-608 sur la simplicité divine. Sur la simplicité chez Pierre de Jean Olivi, voir J. Toivanen, Perception and the Internal Senses. Peter of John Olivi on the Cognitive Functions of the Sensitive Soul, Leyde - Boston, Brill, 2013, p. 30-38; J. F. Silva, J. Toivanen, «The Active Nature of the Soul in Sense Perception: Robert Kilwardby and Peter Olivi», Vivarium, t. XLVIII, 2010, p. 264-266. Cependant, ni l'article de Toivanen ni celui de Silva ne traite de la simplicité divine, qui est d'une grande importance pour notre sujet.

23. Olivi, Quaestiones in secundum librum..., II, q. 33, vol. I, p. 607-609.

24. Ibid., II, q. 13, vol. I, p. 231-255 ("an universalia sint in individuis suis secundum suam universalitatem an tantum in intellectu»). Après avoir présenté deux positions réalistes, Olivi propose son propre conceptualisme: «Tertii sunt qui volunt quod universalia nullam habeant universalitatem nisi solum in intellectu et secundum intellectum, it quod nihil sit in rebus extra intellectum aliquo modo nisi particulare et individuatum [...] Iste autem modus dicendi solemnior est et communior et verior, prout credo", ibid., p. 235. 
le problème est le suivant: les formes substantielles sont-elles susceptibles du plus et du moins? Olivi distingue une possible réponse logique d'une réponse métaphysique: dans une perspective logique, les formes substantielles comme "homme» n'acceptent pas le plus et le moins, le concept «homme» signifiant uniquement ce qu'il y a de commun à Pierre, Marie, Jean, etc. Dès lors, on ne peut dire que Pierre est plus homme que Marie ${ }^{25}$. Cette simplicité logique des formes substantielles renvoie à la thèse olivienne déjà évoquée selon laquelle une species est présente tout entière (tota), mais non totaliter, dans les individus qui lui correspondent. Cela est vrai uniquement parce que chaque individu inclus sous une espèce peut être appelé «homme» ou «âne», et non parce que la species universelle existerait réellement dans ces particuliers concrets.

Mais en un sens métaphysique, les formes substantielles sont en revanche susceptibles du plus et du moins. Pierre de Jean Olivi rejette ainsi la position aristotélicienne des Catégories selon laquelle une substance n'accepte pas le plus et le moins ${ }^{26}$. Franciscus de Marchia fera de même une génération plus tard (au moins pour ce qui est de l'âme intellective $)^{27}$. On peut dire qu'un pur sang est plus un cheval qu'un percheron, ou qu'un grand feu est plus un feu qu'un petit feu. Pourquoi parlons-nous ainsi? Pour Olivi, ce ne peut être la matière particulière qui est informée par la forme substantielle en question, car la matière ne contribue pas à l'essence de la forme. Selon lui, l'opinion selon laquelle la matière accidentelle est la raison du plus et du moins de la forme substantielle est « ridicule $»^{28}$. C'est bien plutôt la forme substantielle qui doit avoir, plus ou moins qu'une autre, ses parties essentielles. Aussi la raison de l'intension et de la rémission des formes substantielles réside-t-elle selon Olivi dans la pluralité ou la grandeur des parties internes de ces formes. S'il n'y avait pas de parties réelles, rien ne pourrait être ajouté ou soustrait des formes substantielles. Dans ce cas, les formes auraient une essence simplement ponctuelle, ou une actualité et une simplicité de type ponctuel. Cela conduirait à des absurdités pour notre auteur: la forme substantielle d'un ange, par exemple, n'aurait qu'une grandeur totalement simple, n'autorisant aucune sorte de pluralité. Chaque parcelle de sa grandeur serait complètement identique aux autres, de sorte qu'il n'y aurait aucune différenciation interne. Mais un ange est un être spirituel, et non une entité quantitative géométrique semblable au point. Or une telle simplicité interne à la grandeur indifférenciée ne peut être

25. Ibid., II, q. 22, vol. I, p. 391-392.

26. Aristote, Catégories, c. 5, 3 b33.

27. Olivi, Quaestiones in secundum librum..., II, q. 22, vol. I, p.393.

28. Ibid., II, q. 22, vol. I, p. 393. 
attribuée à aucune créature, mais est réservée à Dieu ${ }^{29}$. Il faut donc conclure qu'inversement, toute créature doit avoir une essence plurielle - ce qui n'est pas le cas de Dieu. Par conséquent, il n'y a aucun fondement à maintenir la conception thomiste d'une âme humaine ontologiquement simple. La thèse d'Olivi est problématique, surtout si nous considérons les formes substantielles des êtres humains - pourquoi une personne devrait-elle être plus un être humain qu'une autre? Il y a là très probablement l'influence des condamnations de 1277 . Olivi a pu vouloir expliquer pourquoi l'anima intellectiva du Christ, une forme substantielle, est plus noble que celle des êtres humains ordinaires ${ }^{30}$. Mais comme nous le verrons plus loin, cette thèse est également essentielle à son éthique, en particulier pour soutenir que l'homme est doté de libre arbitre.

Il est vrai que pour Thomas d'Aquin, toutes les créatures ne sont pas simples, puisqu'elles sont composées d'une essence et d'un être ${ }^{31}$. Mais au moins en un sens métaphysique, l'essence ou forme substantielle de l'être humain est simple, et n'est diverse que si l'on considère les fonctions ou facultés (virtutes) de l'âme. Au contraire, pour Olivi tous les types d'essence - hormis celle de Dieu - possèdent formellement des parties essentielles distinctes. Comme nous le verrons, cela est également vrai pour les humains.

Olivi donne un second argument en faveur de la différenciation interne des formes substantielles: selon Thomas d'Aquin, la forme essentielle d'une créature est simple et n'a aucune partie vraiment distincte. Elle doit pourtant avoir une grandeur limitée puisqu'elle est une créature. Cependant, pour Olivi, il n'y a aucune raison pour que cette forme essentielle ne puisse avoir une grandeur infinie, puisque la simplicité n'est pas contraire à l'infinité selon Thomas d'Aquin. À nouveau une telle créature serait semblable à Dieu $^{32}$.

En outre, une telle forme substantielle simple serait complètement autosuffisante et absolue. Aussi n'informerait-elle aucune matière. Toute forme accueillie dans une matière doit être constituée de parties, puisque

29. Olivi, Quaestiones in secundum librum..., II, q. 22, vol. I, p. 401.

30. Cf. note 27 .

31. Thomas d'Aquin, De ente et essentia, c. IV, Editio Leonina, t. XLIII, Rome, Editori di San Tommaso, 1976, p. 376b: «Omnis autem essentia uel quiditas potest intelligi sine hoc quod aliquid intelligatur de esse suo: possum enim intelligere quid est homo uel fenix, et tamen ignorare an esse habeat in rerum natura; ergo patet quod esse est aliud ab essentia uel quiditate».

32. Olivi, Quaestiones in secundum librum..., II, q. 22, vol. I, p. 401: "Praeterea, qua ratione idem simplicissimum potest habere tantam magnitudinem, eadem ratione poterit habere et infinitam secundum illam consequentiam quae a multis infertur: quod qua ratione aliquid potest esse in duobus locis, eadem ratione et infinitis; quia non plus repugnant ei infinita loca quam duo, immo per id ipsum repugnant duo et infinita, sicut suo loco est ostensum ». 
la matière doit pouvoir porter cette forme. Il doit y avoir une adéquation mutuelle de la matière et de la forme, qui ne peut être réalisée si les parties de la forme ne sont pas proportionnées aux parties de la matière. Ainsi, une partie de la forme informe une partie de la matière. Si la forme existait dans chaque partie de la matière totum et totaliter, alors la forme tout entière existerait plusieurs fois dans le tout de la matière. Par exemple, la forme entière d'un animal existerait plusieurs fois dans le corps d'un animal: une fois dans la tête, une fois dans une jambe, etc. Ainsi y aurait-il plusieurs animaux entiers dans un seul corps animal ${ }^{33}$. Avec cet argument, Olivi rejette implicitement le modèle thomiste d'une inhérence des formes substantielles dans la matière. Pour Thomas d'Aquin la relation formematière est une correspondance entre quelque chose d'ontologiquement simple dénuée de distinctions réelles (à savoir la forme ou l'essence), et une diversité corporelle quantitative et extensive. Au contraire, Olivi conçoit l'hylémorphisme comme une relation entre deux sortes de pluralité ou de grandeur: une forme plurielle avec des parties formellement distinctes (dans le cas de l'être humain) ou une essence dotée de grandeur intensive, et une matière constituée de parties. La forme et la matière ne peuvent être mises en relation que parce qu'elles ont une structure interne similaire - toutes deux ont des parties. Une forme substantielle doit informer un corps ou sa matière partie par partie, et non tota et totaliter in parte. Olivi réserve le modèle holenmériste de Thomas d'Aquin exclusivement à la présence de Dieu dans le monde; seul Dieu a une simplicité qui lui permet d'être totaliter présent en chaque partie du monde. Les autres types de présence, créés, n'exigent pas ce concept fort de totalité. Si d'autres formes substantielles étaient présentes dans les parties matérielles à la manière dont Dieu l'est au monde, c'est-à-dire totum et totaliter, nous aurions une pluralité de substances complètes correspondant à la pluralité des parties matérielles, et rien n'empêcherait que ces formes n'informent des amas de matière en nombre infini. Dans ce cas, les âmes humaines ou les autres formes substantielles posséderaient un degré suprême de simplicité combiné à l'infinité, chose strictement réservée à Dieu ${ }^{34}$.

Selon Pierre de Jean Olivi, le problème de cette conception thomiste est son concept trop fort de simplicité ontologique et son articulation à celui d'infinité. Quel serait le mode opératoire d'une forme présentant une telle simplicité? Une telle forme substantielle produirait chacune de ses différentes opérations et activités en totalité, comme un tout. Autrement dit, chaque partie de cette forme ne pourrait produire que l'opération complète.

33. Ibid., II, q. 22, vol. I, p. 401-402.

34. Ibid., II, q. 22, vol. I, p. 401. 
Une seule partie de la forme aurait le même effet que la forme entière. Mais derechef, ce mode opératoire totum et totaliter n'est possible que pour Dieu, car selon Olivi, les créatures opèrent selon une partie particulière de leur essence, et non selon toute leur forme substantielle. Olivi soutient un modèle agrégatif de causalité, ainsi que des propriétés substantielles d'une substance. Il l'emprunte aux agrégats physiques matériels relativement simples que sont par exemple le feu ou la terre: dans ce cas, nous pouvons voir qu'une unique partie de terre, pour prendre cet exemple, a moins d'effets que l'agrégation d'une multitude de parties, et que ses propriétés accidentelles (comme le poids) sont affaiblies. Dès lors, si un trait accidentel comme le poids est renforcé par une telle agrégation, a fortiori la forme substantielle de cet agrégat est plus forte que celle de chacune de ses parties ${ }^{35}$.

Thomas d'Aquin privilégie dans sa psychologie la relation un-multiple: une forme substantielle ontologiquement simple informe une matière organique et constitue l'être et la forme des parties du corps. Olivi critique cette image, réservant strictement le modèle de l'Aquinate à la présence de Dieu dans le monde. Seul Dieu est totalement présent dans chaque partie de l'univers selon une totalitas essentiae. Toutes les autres créatures n'ont pas une telle forme substantielle simple, leur essence est susceptible du plus et du moins, et leur mode de présence dans la matière est une relation de partie à partie. À l'inverse de Thomas d'Aquin (qui considère que, pour son hylémorphisme, la totalité est, au moins selon l'essence, un concept pertinent), Olivi exclut complètement tout type de totalité du mode de présence de la forme créée à sa matière et aux parties qui lui sont liées. Le contraire conduirait à une plurilocation de la forme semblable à celle de Dieu.

Que répondrait Thomas d'Aquin à la critique de Pierre de Olivi? Dans la Somme de théologie (I, q. 76, a. 8), il traite d'un argument similaire: «Dans le second livre du De anima, Aristote écrit: comme la partie de l'âme est relative à la partie du corps, l'âme entière est liée à tout le corps animé. Mais si l'âme entière existe dans chaque partie du corps, alors il s'ensuit que chaque partie du corps est un animal ${ }^{36}$. Cette objection semble très proche de la ligne d'argumentation olivienne, bien que ce passage ne traite pas du problème de la simplicité d'une forme substantielle. La réponse de Thomas d'Aquin commence par une définition: un animal est ce qui

35. Olivi, Quaestiones in secundum librum..., II, q. 22, vol. I, p. 402.

36. Thomas d'Aquin, Summa theologiae..., I, q. 76, a. 8, p. 232a: «Praeterea, in II De anima dicitur quod sicut se habet pars animae ad partem corporis, ut visus ad pupillam, ita anima tota ad totum corpus animalis. Si igitur tota anima est in qualibet parte corporis, sequitur quod quaelibet pars corporis sit animal». 
est composé d'une âme et d'un corps entier, proportionné à cette âme et perfectionné par elle. Dans cette perspective, c'est-à-dire comme quelque chose qui est lié au corps entier et qui le perfectionne, l'âme n'existe pas dans chaque partie du corps. Par conséquent, pour Thomas d'Aquin, on ne peut appeler une partie d'animal un animal ${ }^{37}$. Il s'agit ici de montrer la relation naturelle ou la proportion de l'âme à tout le corps. Ce n'est pas comme si l'âme et le corps étaient deux entités distinctes, et que l'on devait déterminer leur relation dans un second temps. En tant que forme et matière, ce sont plutôt les deux aspects ou les deux principes explicatifs d'une seule et même entité. Leur unité est plus forte que celle de deux choses distinctes, les deux formant un unum simpliciter ${ }^{38}$. Aussi convaincant que semble ce modèle, reste un léger problème: ce mode d'argumentation est valable pour les plantes et les animaux, mais des difficultés surgissent pour les êtres humains, qui sont informées par une âme intellective immatérielle capable de penser et d'exister après la corruption du corps. Ici, le modèle des deux aspects d'une même entité et du caractère étroitement proportionné de la forme à la matière est à tout le moins problématique. Nous verrons que la psychologie de Pierre de Jean Olivi a moins de problèmes de ce type, car depuis le début il confère à l'âme intellective un statut ontologique spécial - naturellement au prix d'une approche plus dualiste.

\section{La version olivienne de l'hylémorphisme de l'âme et du corps}

Bien que Pierre de Jean Olivi emploie, comme Thomas d'Aquin, les notions de matière et de forme, il le fait d'une manière complètement différente: pour Thomas d'Aquin, la matière et la forme des êtres vivants forment un unum simpliciter, une unité indivisible. Si une main était séparée d'un être humain, ce ne serait plus une main vivante et une partie de cet organisme ${ }^{39}$. C'est précisément la raison pour laquelle, pour Thomas d'Aquin, la forme substantielle d'un être vivant existe comme un tout dans chaque parcelle de matière. La forme substantielle d'un être vivant constitue l'existence de

37. Thomas d'Aquin, Summa theologiae..., I, q. 76, a. 8, ad 3, p. 233 b.

38. Pour l'arrière-plan aristotélicien, voir Aristote, De anima II 1, 412b6-8. Cf. P. Rubini, Pietro Pomponazzis Erkenntnistheorie. Naturalisierung des menschlichen Geistes im Spätaristotelismus, Leyde, Brill, 2015, p. 94-97.

39. Thomas d'Aquin, Quaestiones disputatae de anima, q. 10, p. 90 sq., I Sent. 24.1.1c, In duodecim libros Metaphysicorum Aristotelis expositio X 3, M. R. Cathala et R. M. Spiazzi (éd.), Turin - Rome, Marietti, 1950, p. 471, note 1974. Cf. R. Pasnau, Thomas Aquinas on Human Nature. A Philosophical Study of Summa theologiae Ia 75-89, Cambridge, Cambridge University Press, 2002, p. 92-93. 
toute la matière et de chacune de ses parties ${ }^{40}$. Pour Olivi, la forme et la matière sont réellement des entités distinctes. Elles ont une existence propre. Si la matière n'était pas vraiment distincte de la forme, elle ne pourrait recevoir l'actualité de la forme ${ }^{41}$. Bien que distinctes, la matière et la forme sont nécessairement corrélées, la matière est orientée vers la forme, et vice versa. Toutes les formes créées doivent être reçues dans quelque chose de vraiment distinct d'elles, sinon elles seraient autosuffisantes et semblables à Dieu - cet argument explique à la fois la distinction réelle de la matière et de la forme, et leur corrélation ${ }^{42}$. Pierre de Jean Olivi souligne explicitement le caractère relationnel du monde et la connexion générale de toutes choses, qui peuvent seulement être expliqués par la matière. Pour qu'il y ait une connexion et une inclination mutuelle des choses, sont requises des puissances passives (c'est-à-dire des morceaux de matière), qui soient capables de recevoir les actions des objets reliés ${ }^{43}$.

Il y a deux sortes de matière, comme il y a deux sortes distinctes de formes: corporelle et spirituelle. Mais la matière spirituelle n'est pas la matière jointe à la différence spécifique «spirituelle». Autrement dit, les deux sortes de matière ne sont pas distinctes du fait de quelque chose de réel ajouté à leur essence. C'est plutôt que la matière peut avoir différentes orientations (rationes respectivae) ou différentes relations à l'égard de différents types de forme. Ce qui distingue la matière spirituelle de la matière corporelle est sa vocation à être informée par une forme spirituelle. Ainsi l'hylémorphisme de Pierre de Jean Olivi est d'un certain point de vue plus strict que celui de Thomas d'Aquin: pour l'Aquinate, une forme spirituelle, comme l'anima intellectiva, peut informer une matière corporelle - le corps humain en l'occurrence. Pour Pierre de Jean Olivi, une forme spirituelle doit informer une matière spirituelle, tandis qu'une forme corporelle ne peut informer qu'une matière corporelle ${ }^{44}$. Par conséquent, Olivi assume la thèse selon laquelle toutes les créatures sont composées de matière et

40. Thomas d'Aquin, Summa theologiae..., I, q. 76, a. 8, p. 232. Cf. Pasnau, Thomas Aquinas on Human Nature..., p. 93-94.

41. Olivi, Sent. II, q. 16, B. Jansen (éd.), vol. I, p. 316. Sur le concept olivien de matière, voir Pierre de Jean Olivi, La Matière, T. Suarez-Nani, C. König-Pralong, O. Ribordy et A. Robiglio (éd.), Paris, Vrin, 2009, et T. Suarez-Nani, «Pierre de Jean Olivi et la subjectivité angélique», Archives d'histoire doctrinale et littéraire du Moyen Âge, t. LXX, 2003, p. 235-245.

42. Olivi, Quaestiones in secundum librum..., II, q. 16, vol. I, p. 320. Cf. T. Suarez-Nani, «Introduction », in Pierre de Jean Olivi, La Matière, p. 40.

43. Olivi, Quaestiones in secundum librum..., II, q. 16, vol. I, p.312-13.

44. Il y a une exception à cette règle: les formes spirituelles inférieures, comme celles des âmes animales, semblent être capables d'informer directement la matière corporelle. Ibid., II, q. 49, B. Jansen (éd.), vol. II, p. 17. 
de forme, ce qui constitue un retour à la thèse d'Ibn Gabirol ${ }^{45}$. La matière spirituelle jouera un rôle important dans la conception de l'âme humaine, comme on le verra plus loin.

Le point de départ de la psychologie d'Olivi est un principe que Thomas d'Aquin refuse: la différenciation fonctionnelle des parties de l'âme implique une distinction ontologique. L'argument de Thomas d'Aquin est que l'âme humaine est une quant à son essence, mais diverse quant à ses fonctions (virtutes). Pierre de Jean Olivi pense qu'il n'y a pas une seule forme substantielle de l'être humain, mais plusieurs: (1) la forme du corps, qui garantit son unité et sa structure, même après la mort. Cet aspect est important pour la vénération des reliques des saints - s'il ne reste rien appartenant à l'être humain qu'était le saint, cette coutume serait dénuée de tout sens. Avec son approche aristotélicienne, Thomas d'Aquin a plus de difficultés à l'expliquer, puisque selon Aristote, une main séparée d'un être humain n'est plus une main humaine stricto sensu: il $s^{\prime}$ agit seulement d'un homonyme ${ }^{46}$. Pour ce qui est de l'âme, Pierre de Jean Olivi distingue les essences ou les parties formelles (partes formales) que sont les âmes (2) végétative, (3) sensitive, et (4) intellective, puisqu'elles sont toutes trois fonctionnellement différentes: l'âme végétative autorise les fonctions de nutrition, de croissance et de reproduction, la sensitive permet la perception et le désir, l'intellective est responsable en l'homme de la pensée et du libre arbitre ${ }^{47}$.

Comme dans le cas de la matière et de la forme, Olivi commence par distinguer essentiellement les formes substantielles de l'être humain. La question est dès lors la suivante: comment expliquer l'unité de la personne? Thomas d'Aquin n'a ici aucun problème, puisqu'il fonde sa réflexion sur la notion d'unum simpliciter. Olivi doit recourir à sa conception de la matière pour résoudre ce problème. L'âme végétative et l'âme sensitive informent toutes deux une matière corporelle, comme les âmes des animaux. L'âme intellective ne peut être présente dans la matière corporelle, puisqu'elle est une forme purement spirituelle. Si l'âme intellective était la forme d'une matière corporelle, soit elle conférerait son incorruptibilité au corps, et le

45. Cf. la traduction latine du XII ${ }^{e}$ siècle de Dominicus Gundissalinus: Avencebrolis (Ibn Gebrol) Fons vitae ex Arabico in Latinum translatus ab Iohanne Hispano et Dominico Gundissalino, C. Baeumker (éd.), Münster, Aschendorff (Beiträge zur Geschichte der Philosophie, 1, H. 2-4), 1892-1895.

46. On trouve une position similaire chez Guillaume d'Ockham. Voir D. Perler, «Ockham über die Seele und ihre Teile», Recherches de théologie et philosophie médiévales, t. LXXVII, $\mathrm{n}^{\circ} 2,2010, \mathrm{p} .313-350$, plus particulièrement p. 321 .

47. Olivi, Quaestiones in secundum librum..., II, q. 51, vol. II, p.181-198. 
corps humain serait immortel, soit l'anima intellectiva deviendrait mortelle et cesserait d'exister avec la corruption du corps ${ }^{48}$.

C'est ici qu'Olivi introduit la matière spirituelle: l'homme ne possède pas seulement un corps - matière corporelle-, mais aussi une matière spirituelle qui peut être actualisée par l'intellect. Mais les âmes sensitive et végétative demeurent séparées de l'intellect. Afin de permettre une unité substantielle de la personne humaine, Olivi soutient que l'anima vegetativa et l'anima sensitiva n'informent pas seulement la matière corporelle, mais aussi la matière spirituelle ${ }^{49}$. L'âme végétative permet à la matière spirituelle d'être informée par l'âme sensitive, et l'âme sensitive dispose cette matière à être informée par l'âme intellective ${ }^{50}$. Jean Olivi nomme cette corrélation de toutes les parties de l'âme et du corps la colligantia potentiarum $^{51}$. Ainsi Olivi, tout en affirmant la distinction réelle entre les trois parties de l'âme, et entre l'âme et le corps, les articule en une unité substantielle au moyen de différentes relations: la relation de l'intellect et de la matière spirituelle, l'information de la matière spirituelle par l'âme sensitive et l'âme végétative, et in fine la relation des âmes sensitives et végétatives à la corporéité. L'unité substantielle de l'être humain est un réseau de relations d'entités distinctes. Tandis que chez Thomas d'Aquin, la pierre angulaire de son anthropologie est l'unum simpliciter, l'ontologiquement un, la conception olivienne de l'être humain s'appuie sur la notion de relation (ratio respectiva). Thomas d'Aquin traite de la même manière les hommes et tous les composés matière-forme: comme des una simpliciter. Jean Olivi cherche à donner à l'hylémorphisme humain

48. Olivi, Quaestiones in secundum librum..., II, q. 51, vol. II, p.104-105; p.115-117.

49. Ibid., II, q. 51 App., vol. II, p. 184.

50. Ibid., Sent. II, q. 51 App., vol. II, p. 193.

51. Cf. A. Krömer, Potenzhierarchie und Dynamismus des Geistes. Ein Beitrag zur Erkenntnismetaphysik des Petrus Johannis Olivi (1248/49-1298) (Diss.), Fribourg, J. Krause, 1974, p. 28-81; E. Bettoni, Le dottrine filosofiche di Pier di Giovanni Olivi, Milan, Vita e pensiero, 1959, p. 333-397 et p. 446-466; T. Schneider, Die Einheit des Menschen. Die anthropologische Formel "anima forma corporis" im sogenannten Korrektorienstreit und bei Petrus Johannis Olivi. Ein Beitrag zur Vorgeschichte des Konzils von Vienne, Münster, Aschendorff, 1973, p. 227-246; F.-X. Putallaz, La Connaissance de soi au XIII siècle. De Matthieu d'Aquasparta à Thierry de Freiberg, Paris, Vrin, 1991, p. 99-102; R. Pasnau, "Olivi on the Metaphysics of Soul», Medieval Philosophy and Theology, t. VI, 1997, p. 109-132; J. Toivanen, Perception and the Internal Senses..., p. 62-75; C. Rode, Zugänge zum Selbst. Innere Erfahrung in Spätmittelalter und Früher Neuzeit, Münster, Aschendorff (Beiträge zur Geschichte der Philosophie und Theologie des Mittelalters), 2015, p.112-115. La conception des colligantia trouve sa source chez Bonaventure. Voir E. Bettoni, «La legge fondamentale della psicologia Bonventuriana: la "colligantia naturalis" ", in San Bonaventura maestro di vita Francescana e di sapienza cristiana (Atti del congresso internazionale per il VII centenario di San Bonvantura da Bagnoregio, Roma, 19-26 septembre 1974), A. Pompei (éd.), vol. II, Rome, Pontificia Facoltà Teologica «San Bonvaventura», 1976, p. 483-491. 
un rang spécial et plus noble que celui des animaux ou des pierres, en introduisant le concept de matière spirituelle.

Deux principes guident la version olivienne de l'hylémorphisme anthropologique en général: (a) seul un type adéquat de forme peut inhérer dans un type correspondant de matière, et (b) la différenciation fonctionnelle implique une distinction essentielle. Ces principes guident également la manière particulière dont l'âme est présente dans le corps, et nous revenons ici à la conception olivienne de la totalitas.

L'âme entière, prise comme l'unité de l'âme végétative, sensitive et intellective, transcende la matière du corps d'une manière différente des formes étendues dénuées de toute matière spirituelle (comme celles des rochers, du feu, ou des âmes simplement animales). Olivi appelle parfois l'âme unifiée anima rationalis, à ne pas confondre avec l'anima intellectiva ${ }^{52}$. Ainsi toute l'âme n'est pas présente dans la matière corporelle selon son ambitus virtuel complet ou selon son domaine d'information, c'est-à-dire selon sa totalitas: la partie intellective n'est pas orientée vers le corps mais vers la matière spirituelle - ici le principe (a) devient effectif. Le domaine de l'âme humaine est plus large que la simple information du corps. Car seules les âmes sensitives et végétatives sont responsables de cette information ${ }^{53}$. Cependant nous pouvons dire qu'en un sens l'âme entière existe dans le corps par une sorte d'existence totale qui ne peut être divisée en différentes parties séparées, et qui ne peut être multipliée dans les membres du corps. Dans cette perspective, toutes les parties du corps sont informées par une seule âme, dès lors que le corps appartient à une species - homme. Selon cette manière de parler, l'âme dans la tête n'est pas éloignée spatialement de l'âme dans le pied ${ }^{54}$.

Cependant, l'âme possède une pluriformité virtuelle et essentielle, une grandeur qui est communiquée au corps différemment selon l'intension et l'extension ${ }^{55}$. Ici, Pierre de Jean Olivi applique les principes (a) et (b): les différentes fonctions de l'âme impliquent la distinction essentielle de leurs parties (b), et les différentes parties formelles de l'âme ne sont reliées qu'aux parties corporelles adéquates et spécifiquement distinctes (a). Les parties corporelles sont spécifiquement distinctes en tant qu'elles transmettent les actions de parties de l'âme elles-mêmes essentiellement distinctes. Ainsi, dans la mesure où l'œil est informé par l'âme sensitive et l'estomac par l'âme végétative, ils appartiennent à deux espèces (species) différentes de matière.

52. Olivi, Quaestiones in secundum librum..., II, q. 49, vol. II, p. 17.

53. Ibid., II, q. 49, vol. II, p. 17.

54. Ibid., p. 19.

55. Ibid. 
En outre, plusieurs organes réellement distincts, comme la main et l'œil, peuvent être informés par une seule et même puissance de l'âme, ici par le sens du toucher. Olivi appelle ces différentes manifestations du sens tactile des aspects ou des modes. Une faculté de l'âme, par exemple la vue, peut même être reçue dans son organe selon des dispositions corporelles variées: une fois dans la rétine, une autre dans le corps vitreux, etc ${ }^{56}$. La relation de l'âme au corps est une fine proportion entre deux pluralités diversement structurées. Dans certains cas, l'unité des parties du corps et des parties de l'âme est déterminée par la nature de l'âme, dans d'autres par la nature du corps et ses dispositions.

En outre, toutes les sortes de matière ne sont pas également informées par l'âme (c'est-à-dire les parties sensitives et végétatives). Pierre de Jean Olivi distingue trois sortes de matière relativement à leur information par l'âme, et trois sortes d'orientation de l'âme vers les différentes espèces (species) de matière: (1) la materia radicalissima, par exemple le cœur et la tête, qui sont indispensables à l'animation du corps, (2) une sorte secondaire de matière comprenant les organes corporels comme la main et l'œil. Olivi appelle aussi cette matière radicalis. Mais un corps pourrait être informé par une âme sans elle. Ces organes sont appropriés par l'âme seulement médiatement, dans la mesure où ils sont reliés à la materia radicalissima. (3) Enfin, il y a une matière nutritive, une nourriture, qui peut être appropriée par l'âme de temps à autre selon les lois biologiques du métabolisme humain. L'âme n'est primordialement et selon la totalité (totalitas) de son ambitus unie qu'à la première sorte de matière - la plus radicale -, c'est-à-dire la tête et le cœur. Elle s'approprie les autres sortes de matière seulement au moyen de la materia radicalissima ${ }^{57}$. Albert le Grand propose un tel mode médiatisé d'information du corps par l'âme dans le De homine, au moins en ce qui concerne l'opération de l'âme dans le corps: l'âme est unie au corps par les media que sont le cœur et les spiritus vitales ${ }^{58}$ - ce parallèle va dans le sens de la thèse de Catherine König-Pralong selon laquelle Pierre de Jean Olivi aurait pu avoir connaissance de l'œuvre d'Albert le Grand ${ }^{59}$.

56. Olivi, Quaestiones in secundum librum..., II, q. 49, vol. II, p.14-15.

57. Ibid., II, q. 49, vol. II, p. 19. Texte parallèle : ibid., II, q. 16, p. 337-338.

58. Albert le Grand, De homine..., p. 571-572. Cf. H. Anzulewicz, «Grundlagen von Individuum und Individualität in der Anthropologie des Albertus Magnus», in Individuum und Individualität im Mittelalter, J. A. Aertsen et A. Speer (éd.), Berlin - New York, De Gruyter (Miscellanea Mediaevalia, 27), 1996, p.124-160.

59. Cf. C. König-Pralong, "Olivi et le formalisme ontologique. Lectures d'Aristote, d'Averroès, et critique d'Albert?», in Pierre de Jean Olivi. Philosophe et théologien (Actes du colloque de philosophie médiévale, 24-25 octobre 2008, Université de Fribourg), C. König-Pralong, O. Ribordy et T. Suarez-Nani (éd.), Berlin - New York, De Gruyter (Scrinium Friburgense, 29), 2010, p.135-165. 
Pour Olivi, il n'y a pas seulement les parties radicales de la matière, mais aussi les formes radicales de l'âme: dans la célèbre question 57 sur le libre arbitre, il décrit quatre degrés d'enracinement dans la matière. De manière surprenante, l'intellect est la plus radicale des parties de l'âme. S'ensuivent l'âme sensitive, puis l'âme végétative, et enfin la forma corporeitatis ${ }^{60}$. Pourquoi l'intellect est-il si profondément enraciné dans la matière? Cette affirmation paraît étrange d'après ce que nous savions de la psychologie d'Olivi jusque-là. En réalité, cela fait parfaitement sens: les formes les plus radicales rendent la matière et tout le composé humain plus stables. Elles pénètrent la potentialité de la matière d'une manière plus complète. Gardons en tête que le philosophe ne parle pas uniquement de la matière corporelle, dont la consistance tient à la pénétration et la stabilisation par des formes plus hautes. Lorsqu'il traite de l'intellect, il fait référence à la matière spirituelle. L'important ici est la direction de la relation entre les formes de l'âme d'une part et la matière d'autre part: bien que dans certains cas, comme vu plus haut, les différentes modalités d'union de l'âme et du corps soient dues à la disposition des parties matérielles, il s'avère dans ce passage que l'impulsion primordiale, ou le pouvoir principal, de configuration provient des formes les plus hautes, dont l'information a été préparée par des formes inférieures. Ainsi l'information du corps et de la matière spirituelle par l'âme humaine est-elle un processus intriqué et complexe, avec de multiples stades intermédiaires de configuration. En un sens, le résultat final - l'information de la matière spirituelle par l'intellect - détermine tout le processus depuis le début.

Pour Thomas d'Aquin, toute l'essence ontologiquement simple de l'âme informe chaque partie du corps. Pour Olivi, l'essence de l'âme n'est pas simple à la manière d'un point ou de Dieu, mais elle comprend des parties essentiellement distinctes. Parmi ces parties, seules l'âme végétative et l'âme sensitive peuvent informer le corps, elles ont une virtus informativa corporis ${ }^{61}$. Même elles cependant ne peuvent dans leur totalité, avec tout leur pouvoir informant, informer chaque partie du corps. Comme nous l'avons $\mathrm{vu}$, elles sont présentes dans le corps seulement selon la nature particulière

60. Olivi, Quaestiones in secundum librum..., II, q. 57, vol. II, p. 335: «Videmus enim quod formae posteriores sunt magis intimatae materiae et magis penetrantes eius potentialitatem quam priores et maiorem dant stabilitatem tam materiae quam toti enti. Unde et habent se ad priores sicut radix ad ramos et sicut principale agens ad instrumentale, et sic semper secundum quod magis ac magis sunt posteriores, sic magis ac magis in istis et multis aliis excedunt, ut verbi gratia, forma mixti formas elementares satisque amplius anima vegetativa excedit ipsam et adhuc amplius sensitiva vegetativam et in sensitiva sensus communis seu tota pars interior sensitivae sensus exteriores ineffabiliusque prae ceteris intellectus praecellit omnes».

61. Ibid., II, q. 49, vol. II, p. 17. 
et la réceptivité des différents organes ou parties du corps, et ce à divers degrés d'intensité. Ici, un autre principe général de l'hylémorphisme olivien entre en ligne de compte: il n'y a aucune forme ontologiquement simple sans quelques parties intensives ou essentielles, et toute présence d'une forme dans la matière a à voir avec une relation de pluralité à pluralité. Cette structure parallèle de la forme et de la matière est valable pour toute forme de relation entre deux entités.

Allons plus loin: cette conception hylémorphique garantit l'affirmation centrale de l'éthique volontariste olivienne, à savoir que nous sommes dotés d'un libre arbitre. Comment s'articulent l'hylémorphisme et la psychologie dans l'éthique de Pierre de Jean Olivi? Un passage de la question 16 du second livre de sa Summa sententiarum le montre clairement: toutes les formes substantielles sont susceptibles du plus et du moins. L'âme intellective de l'être humain est une telle forme substantielle. Ce n'est que là où il peut y avoir intension et rémission qu'il peut y avoir mouvement. Pour Aristote, selon l'interprétation qu'en donne Jean Olivi, l'intellect, comme forme simple et impassible, ne peut être susceptible de mouvement au sens ordinaire du terme, puisqu'il n'a ni matière ni organe ${ }^{62}$. L'automotricité au sens strict - celle de l'intellect ou de n'importe quel objet - est également impossible pour Aristote, la même chose ne pouvant, sous le même rapport, être motrice et mue ${ }^{63}$. Au contraire, pour Pierre de Jean Olivi, le mouvement, et plus particulièrement l'automotricité, est la notion centrale de son éthique. L'automotricité implique la liberté de la volonté, car elle nous permet de réfléchir à nos intentions et souhaits immédiats, et de nous maîtriser ${ }^{64}$. Si la forme intellective ne permettait pas le mouvement, l'automotricité et la liberté de la volonté seraient inaccessibles pour nous, êtres humains.

La conception olivienne de la forme de l'âme intellective est différente de celle d'Aristote et de Thomas d'Aquin: comme déjà souligné, l'intellect n'est pas complètement simple, mais consiste en une grandeur intensive. En tant que tel, il informe la matière spirituelle partie par partie, et non

62. Olivi, Quaestiones in secundum librum..., II, q. 16, vol. I, p. 346-347; cf. Aristote, Metaph. XII 1-2, 1069b3-34. Cf. Aristote, Physique I 4, 407b27-408a18 pour le rejet aristotélicien de la composition au sein de l'intellect. Pierre de Jean Olivi rétorque que sans composition, il ne pourrait pas y avoir plus ou moins d'intellect, et par conséquent il n'y aurait ni mouvement ni augmentation de celui-ci. Aristote admet cependant que l'intellect puisse initier un changement en un sens différent, d'une manière immatérielle et non physique. Cf. Aristote, De anima II 5, 417b6-16. Mais l'argument de Pierre de Jean Olivi est que le changement, l'altération et le mouvement sont des concepts univoques qui doivent s'appliquer de la même manière aux domaines physique et intellectuel.

63. Aristote, Physica VIII 5, 256a4-258b9.

64. Olivi, Quaestiones in secundum librum..., II, q. 57, vol. II, p. 324-325. 
comme un tout dans chaque partie de la matière spirituelle. Dès lors il peut y avoir une augmentation ou un élargissement de la volonté, laquelle appartient à la partie intellective de l'âme. Pierre de Jean Olivi s'appuie ici sur notre expérience: nous pouvons percevoir que grâce aux affections moralement bonnes, notre volonté est élevée, élargie, et devient plus réfléchie. Par conséquent, notre intellect est bien susceptible du plus et du moins ${ }^{65}$. Ainsi l'éthique olivienne se construit-elle sur les affirmations ontologiques très générales de son hylémorphisme et de son anthropologie. Ces affirmations - la rémission et l'intension des formes substantielles et spirituelles - s'opposent fortement à l'ontologie et à la psychologie aristotélicienne de Thomas d'Aquin.

\section{Conclusion}

Les arguments de Pierre de Jean Olivi contre Thomas d'Aquin sur la notion de totalité montrent qu'il conçoit le corps et l'âme non pas comme deux aspects ou deux principes explicatifs d'une seule et même chose, mais comme une composition relationnelle de parties possédant une certaine subsistance. Tandis que Thomas d'Aquin insiste sur l'unité ontologique, Pierre de Jean Olivi met en valeur la structure relationnelle de l'être humain. Alors que l'Aquinate voit l'information du corps par l'âme comme une relation de l'un au multiple, Pierre de Jean Olivi choisit le modèle d'une relation du multiple au multiple. Comme nous l'avons vu, cette décision a aussi un impact sur les principales thèses de son éthique. Robert Pasnau a parlé de l'hylémorphisme psychologique de Pierre de Jean Olivi comme d'un "conte métaphysique " ${ }^{6}$. Il faut réviser ce jugement: Pierre de Jean Olivi prend très au sérieux le présupposé médiéval selon lequel l'intellect est séparable du corps, autant qu'il prend au sérieux la thèse assez intuitive selon laquelle le corps a une certaine structure ou unité, même quand il n'est pas animé. Chez Thomas d'Aquin, il y a une certaine tension entre les deux affirmations selon laquelle l'âme et le corps forment une simple unité d'une part, et celle selon laquelle l'intellect est séparable. Pierre de Jean Olivi essaie de résoudre cette tension au prix de l'introduction d'entités intermédiaires comme la materia spiritualis. Il n’est pas si aisé de déterminer quelle solution a le plus d'inconvénients, au moins au sein du contexte

65. Ibid., II, q. 16, vol. I, p.346. Le concept de latitude (latitudo) de la volonté joue un rôle important dans cette question de la liberté humaine. Cf. Olivi, Quaestiones in secundum librum..., II, q. 57, vol. II, p. 330 : «In appetendo enim et in amando tantum habet mens altitudinem et latitudinem ".

66. Cf. R. Pasnau, «Olivi on the Metaphysics of the Soul...», p. 125. 
théologique et philosophique médiéval. En outre, comme Alain Boureau l'a montré ${ }^{67}$, Pierre de Jean Olivi défend plus largement cette ontologie relationnelle dans son œuvre entière. Il confère aux relations un statut au moins aussi important qu'aux choses concrètes. Loin d'être un "conte», l'hylémorphisme psychologique de Jean Olivi offre une alternative au problème de l'âme et du corps. Cette solution a quelques avantages sur celle de Thomas d'Aquin dans les coordonnées du problème au XIII ${ }^{e}$ siècle, et elle demeure attrayante, au moins dans la mesure où elle traite des hommes différemment des autres substances physiques, en renforçant le rôle des relations.

Christian Rode

Université de Bonn / Würzburg

67. Cf. A. Boureau, «Le concept de relation chez Pierre de Jean Olivi», in Pierre de Jean Olivi (1248-1298). Pensée scolastique, dissidence spirituelle et société, A. Boureau et S. Piron (éd.), Paris, Vrin, 1999, p. 41-55. Également R. Schönberger, Relation als Vergleich. Die Relationstheorie des Johannes Buridan im Kontext seines Denkens und der Scholastik, Leyde - New York - Cologne, Brill, 1994, p.143-150. Sur la relation dans le contexte de l'angélologie: T. Suarez-Nani, «Pierre de Jean Olivi et la subjectivité angélique», Archives d'histoire doctrinale et littéraire du Moyen Âge, t. LXX, 2003, p. 274. 